\title{
Treatment strategies and predicting prognoses in elderly patients with breast cancer
}

This article was published in the following Dove Press journal:

Cancer Management and Research

\author{
Zhi Wang ${ }^{1,2, *}$ \\ Zhangiian Zhou ${ }^{1, *}$ \\ Wenxing $\mathrm{Li}^{\prime}$ \\ Wei Wang ${ }^{3}$ \\ Xin Xie ${ }^{1}$ \\ Jincheng Liu \\ Yongchun Song' \\ Chengxue Dang' \\ Hao Zhang'
}

'Division of Surgical Oncology, The First Affiliated Hospital, Xi'an Jiaotong University, Xi'an, Shaanxi, People's Republic of China; ${ }^{2}$ Division of Surgery, Shaanxi Tuberculosis Hospital, Changan District, Xi'an, Shaanxi, People's Republic of China; ${ }^{3}$ Division of Gynaecology and Obstetrics, The First Affiliated Hospital, Xi'an Jiaotong University, Xi'an, Shaanxi, People's Republic of China

*These authors contributed equally to this work
Correspondence: Hao Zhang

Division of Surgical Oncology, The First Affiliated Hospital, Xi'an Jiaotong

University, 277 West Yanta Road, Xi'an

7I006I, Shaanxi, People's Republic of

China

Tel +86298532 46I0

Email hao.zhang@mail.xjtu.edu.cn
Objective: The prevalence of breast cancer in elderly women (older than 80 years) is expected to rise more dramatically than its incidence. In this study, we evaluated the evidence for treatment guidelines for elderly breast cancer patients.

Patients and methods: All included patients were enrolled from 2010 to 2013 from the Surveillance, Epidemiology, and End Results (SEER) database. The Akaike information criterion (AIC) and Harrell's $C$ statistic were used to perform comparisons. In addition, a propensity score analysis was used to avoid bias caused by data selection criteria. Prognostic factors were selected as nomogram parameters to develop a model to predict survival.

Results: A total of 16998 patients included in the SEER database from 2010 to 2013 had breast cancer and fulfilled the study criteria. Of whom, 13007 patients underwent surgery. Overall survival and cancer-specific survival were significantly better in patients who underwent surgery and/or radiotherapy than in those who did not $(P<0.001)$. In addition, a nomogram system with a $C$ index of 0.83 and an AIC index of 11112.85 was better able to predict prognoses and estimate cancer-specific survival in elderly patients with breast cancer.

Conclusion: A localized surgical approach might provide better results in elderly breast cancer patients. However, radiotherapy improved cancer-specific survival and overall survival in these patients. In addition, a prognostic nomogram directly quantified patient risk by accounting for various prognostic factors without forming risk groups and was better able to estimate cancerspecific survival.

Keywords: breast cancer, elderly patients, survival, prediction, nomogram

\section{Introduction}

Breast cancer is currently the most common cancer in female patients. The prevalence of breast cancer in elderly women (older than 80 years) is expected to rise even more dramatically than its incidence. The average age for a diagnosis of breast cancer is 61 years, and most breast cancer-related deaths occur in patients with an average age of 65 years or older. ${ }^{1}$ A study has shown that there are many differences in the prevalence of strategies used to treat breast cancer between younger and older women. ${ }^{2}$ However, most studies have focused on younger patients. There is a lack of strong evidence for any specific treatment guidelines in elderly patients. The relevant prognostic factors, the best surgical approach and the most suitable treatment strategy for elderly patients with breast cancer remain unclear. In particular, in patients older than 80 years, the influences of various kinds of factors on breast cancer, and specifically on breast cancer-related mortality, remain unknown. ${ }^{3}$ Age is a clinically important prognostic 
factor when determining which treatment modality to use and when estimating overall survival, and it may influence which treatment strategy is ultimately chosen. The choice of surgical approach and subsequent treatment strategies remains controversial in these patients. This is especially important because the majority of elderly patients die because of nonbreast cancer-related reasons. ${ }^{4}$

In this study, we first investigated the clinicopathological characteristics of elderly patients with breast cancer. Second, we attempted to identify the prognostic factors (including clinical and pathological factors) that will be most useful to clinicians making treatment decisions. Finally, we built a nomogram-derived overall survival estimation system that can be used to provide more accurate predictions of overall survival and cancer-specific survival.

\section{Patients and methods}

\section{Patients}

The data collected included patient demographic information, pathological information, clinical procedures and cancerspecific survival in patients with breast cancer. All data collected into the Surveillance, Epidemiology, and End Results (SEER) database from 2010 to 2013 were included to avoid bias caused by changes in treatment strategies over time. The definition of "local" and "radical" surgical approaches is in the Appendix C document in the SEER website. The local approach includes partial mastectomy and subcutaneous mastectomy. The radical approach includes total mastectomy, modified radical mastectomy, radical mastectomy and extended radical mastectomy. The following inclusion criteria were used: 1) patients with pathologically diagnosed breast cancer; 2) patients who were older than 80 years; 3 ) the exact pathological details were accessible and 4) cancer-specific survival was available. In our study, a signed SEER research data agreement form was provided to the SEER program and we were given approval to access and analyze SEER database, which is a public database that does not require ethical approval.

\section{Statistical analyses}

Continuous data are presented as the mean \pm SD. Categorical variables were grouped and compared using the $\chi^{2}$ test or Fisher's exact test. Continuous variables were compared using Student's $t$-test. Univariate and multivariate survival curves were generated using the Kaplan-Meier method, and the differences between curves were analyzed using logrank tests. Univariate and multivariate Cox proportional hazard models were used to identify factors that are associated with cancer-specific survival. The cancer-specific survival in the SEER database was defined as the cause of death. It means that patients were dead because of breast cancer, not other diseases. The prognostic factors were selected as nomogram parameters. Akaike information criterion (AIC) and Harrell's $C$ statistic were used to estimate the proportion of correct predictions and relative discriminative abilities. To adjust the comparisons and avoid distortions from bias in retrospective trials, a propensity score analysis was used. ${ }^{5,6}$ All statistical tests were two sided, and $P$ values $<0.05$ were considered to be statistically significant. Statistical analyses were performed using SPSS 13.0 and R software version 3.3.0 (http://www.rproject.org) with the "SEERaBomb", "rms", "MatchIt", "PSAgraphics" and "AICcmodavg" packages.

\section{Results}

A total of 16998 patients with breast cancer who were included in the SEER database from 2010 to 2013 fulfilled the study criteria. Among these patients, 13007 underwent surgery. The demographic and pathological information for all patients are presented in Table 1 . These data represent all patients, including those with and without surgery. Overall survival and cancer-specific survival were significantly better in patients who underwent surgery than in those who did not $(P<0.001$; Figure $\mathrm{S} 1 \mathrm{~A}$ and $\mathrm{S} 1 \mathrm{~B})$. In addition, overall survival and cancer-specific survival were significantly better in patients who received radiotherapy than in those who did not $(P<0.001$; Figure S1C and S1D).

For patients who underwent a surgical procedure (including partial mastectomy, subcutaneous mastectomy, simply total mastectomy, modified radical mastectomy, radical mastectomy and extended radical mastectomy) with exact pathological information, the detailed demographic and pathological information are presented in Table 2 . In patients older than 80 years, the patient's age at diagnosis was a factor that affected overall survival $(P<0.001$; Figure 1$)$. The risk of a cancer-specific modality dramatically increased as patients get older. The cancer-specific 3-year survival rate was $79 \%$ in patients older than 95 years and $94 \%$ in those between 80 and 85 years. A univariate analysis of overall survival indicated that patient race, the size of the breast cancer and its histological grade, the status of distant metastasis, the surgical approach, the use of radiotherapy, estrogen receptor (ER) positivity, progesterone receptor (PR) positivity, human epidermal growth factor receptor-2 (HER-2) positivity and the status of metastatic axillary lymph nodes were prognostic factors (Figure 1). After a Cox proportional hazard analysis was performed, the first nine of these factors were found 
Table I Demographic, pathological information and cancer-specific survival of all patients

\begin{tabular}{|c|c|c|c|c|}
\hline & \multirow[t]{2}{*}{$\mathbf{N}$} & \multirow[t]{2}{*}{ Percentage (\%) } & \multicolumn{2}{|l|}{ Univariate } \\
\hline & & & Cancer-specific 3-year survival (\%) & $P$-value \\
\hline \multicolumn{5}{|l|}{ Age (years) } \\
\hline Mean (SD) & $84.79 \pm 4.02$ & & & \\
\hline Median (range) & $84(80-108)$ & & & \\
\hline $80-85$ & 10721 & 63.1 & 89 & \\
\hline $85-90$ & 4575 & 26.9 & 84 & \\
\hline $90-95$ & 1429 & 8.4 & 77 & \\
\hline More than 95 & 273 & 1.6 & 69 & $<0.001$ \\
\hline \multicolumn{5}{|l|}{ Race } \\
\hline White & 14523 & 85.4 & 86 & \\
\hline Black & 1357 & 8 & 81 & \\
\hline Others & 1118 & 6.6 & 89 & $<0.001$ \\
\hline \multicolumn{5}{|l|}{ Surgery } \\
\hline Yes & 14030 & 82.5 & 92 & \\
\hline No & 2968 & 17.5 & 60 & $<0.001$ \\
\hline \multicolumn{5}{|l|}{ Radiotherapy } \\
\hline Yes & 4912 & 28.9 & 92 & \\
\hline No & $|165|$ & 68.5 & 83 & \\
\hline Unknown & 435 & 2.6 & 89 & $<0.001$ \\
\hline \multicolumn{5}{|l|}{ ER status } \\
\hline Positive & 14599 & 85.9 & 89 & \\
\hline Negative & 2393 & 14.1 & 69 & \\
\hline Border & 6 & 0.03 & 40 & $<0.001$ \\
\hline \multicolumn{5}{|l|}{ PR status } \\
\hline Positive & 12433 & 73.1 & 90 & \\
\hline Negative & 4526 & 26.6 & 75 & \\
\hline Border & 39 & 0.2 & 61 & $<0.001$ \\
\hline \multicolumn{5}{|l|}{ HER-2 status } \\
\hline Positive & 1790 & 10.5 & 78 & \\
\hline Negative & 14732 & 86.7 & 87 & \\
\hline Border & 476 & 2.8 & 79 & $<0.001$ \\
\hline
\end{tabular}

Abbreviations: ER, estrogen receptor; PR, progesterone receptor; HER-2, human epidermal growth factor receptor-2.

to be independent prognostic factors. These independent prognostic factors were therefore included in the nomogram model. In the nomogram estimation system, each factor from the multivariate Cox proportional hazard regression model was attributed a weighted point value that implied its contribution to a prognosis of survival. We found that elderly breast cancer patients with higher scores had a worse cancer-specific prognosis than was observed in those with lower scores. The final nomogram model that was developed to predict cancer-specific survival in elderly breast cancer patients is shown in Figure 2A.

The predictive accuracy of the final nomogram system was determined by calculating Harrell's $C$ index and AIC index. For the nomogram system, the $C$ index was 0.83 , which is higher than the value of 0.7 expected for a system with accurate overall survival prediction. In addition, it was also higher than the $C$ index for the traditional American
Joint Committee on Cancer (AJCC) staging scheme (0.797). The calibration plot of the nomogram for cancer-specific survival is shown in Figure 2B. From the calibration curve, we found that predicted cancer-specific survival was closely associated with actual cancer-specific survival, with which it was always within a $10 \%$ margin of error. To avoid overfitting the nomogram-derived prognostic model, we also determined its AIC index. The AIC index of the nomogram scheme was 11112.85, which is lower than the AIC index for the AJCC breast cancer staging scheme (11393.13). This indicates that the nomogram system constructed in this study is a better prognostic predictor when estimating cancer-specific survival in elderly patients with breast cancer.

In the nomogram predictive system, we found that tumor size was a strong prognostic indicator of cancer-specific survival. A stratification analysis was performed to evaluate cancer-specific survival in groups that were treated using 


\section{A}

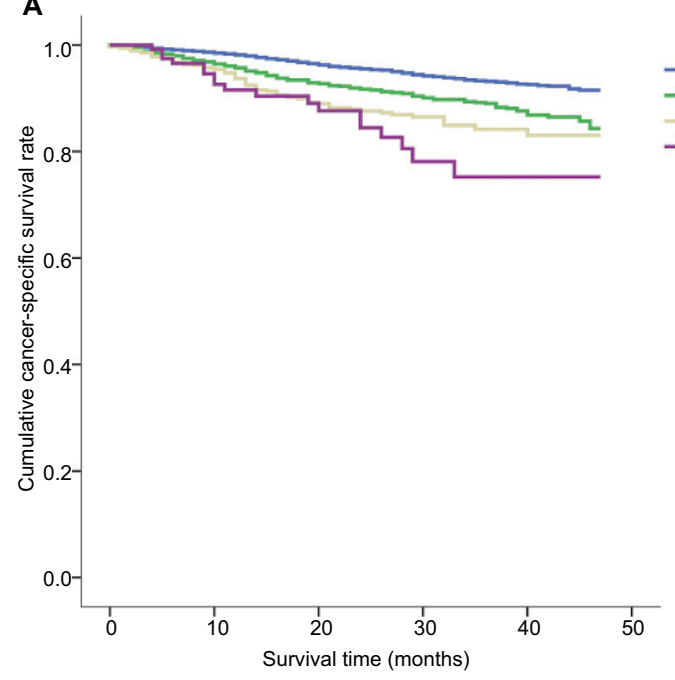

C

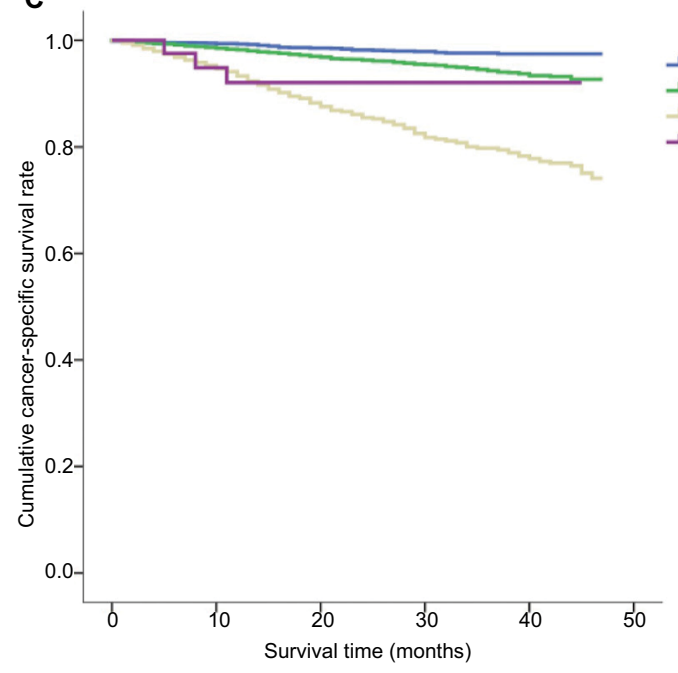

E

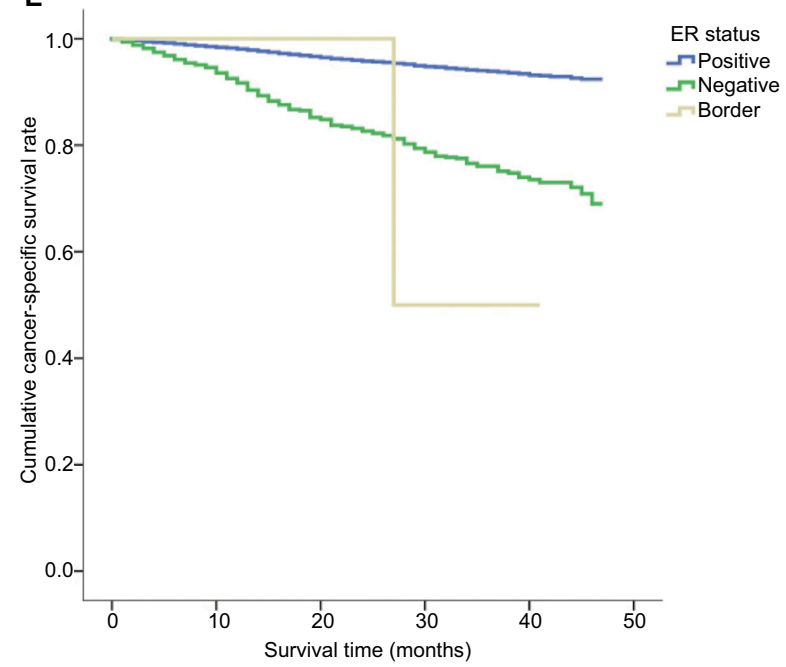

\section{B}

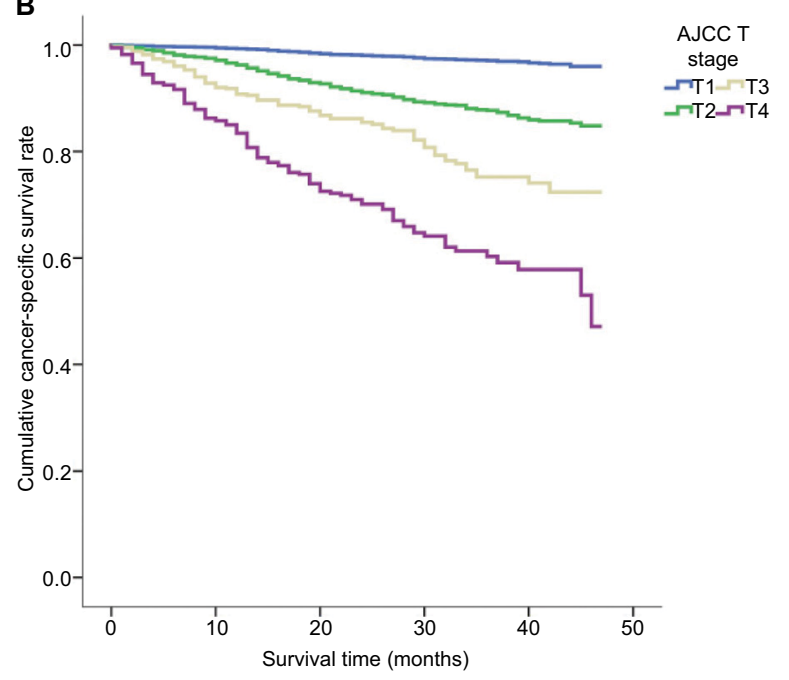

D

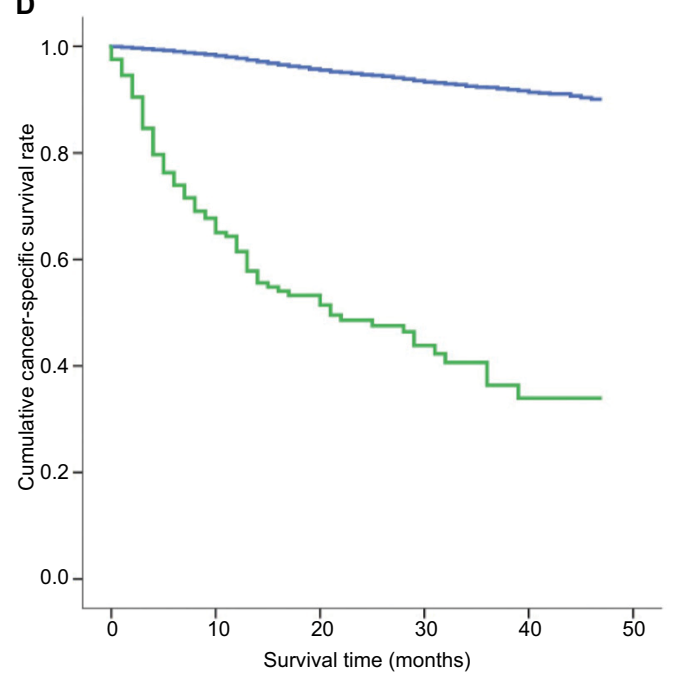

$\mathbf{F}$

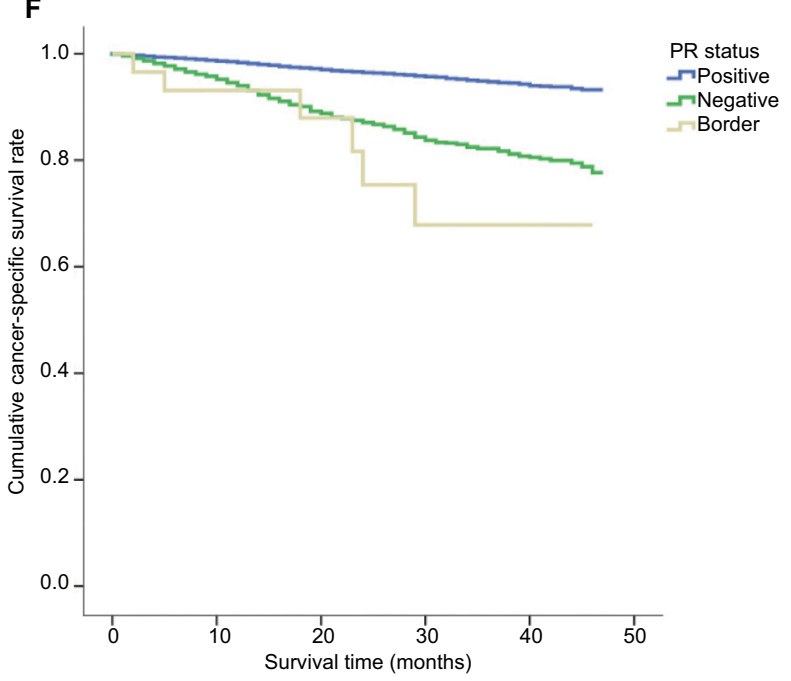

Figure I The independent prognostic factors for cancer-specific survival in elderly breast cancer patients who underwent surgery.

Notes: (A) Kaplan-Meier survival curve for patients of different ages. (B) Kaplan-Meier survival curve for tumors of different sizes. (C) Kaplan-Meier survival curve for tumors of different histological grades. (D) Kaplan-Meier survival curve for patients based on whether they exhibited distant metastasis. (E) Kaplan-Meier survival curve for patients with different ER statuses. (F) Kaplan-Meier survival curve for patients with different PR statuses.

Abbreviations: AJCC, American Joint Committee on Cancer; ER, estrogen receptor; PR, progesterone receptor. 
Table 2 Demographic, pathological information and cancer-specific survival of patients who underwent surgery

\begin{tabular}{|c|c|c|c|c|c|c|}
\hline & \multirow[t]{2}{*}{$\mathbf{N}$} & \multirow[t]{2}{*}{ Percentage } & \multicolumn{2}{|l|}{ Univariate } & \multicolumn{2}{|c|}{ Multivariate } \\
\hline & & & $\begin{array}{l}\text { Cancer-specific } \\
\text { 3-year survival (\%) }\end{array}$ & $P$-value & $P$-value & HR \\
\hline \multicolumn{7}{|l|}{ Age (years) } \\
\hline Mean (SD) & $84.4 I \pm 3.75$ & & & & & \\
\hline Median (range) & $84(80-108)$ & & & & & \\
\hline $80-85$ & 8690 & 66.8 & 94 & & & \\
\hline $85-90$ & 3314 & 25.5 & 89 & & & \\
\hline $90-95$ & 873 & 6.7 & 85 & & & \\
\hline More than 95 & 130 & 1 & 79 & $<0.001$ & $<0.001$ & $1.262-1.534$ \\
\hline \multicolumn{7}{|l|}{ Race } \\
\hline White & 11224 & 86.3 & 92 & & & \\
\hline Black & 931 & 7.2 & 89 & & & \\
\hline Others & 852 & 6.6 & 94 & 0.004 & 0.024 & $0.725-0.978$ \\
\hline \multicolumn{7}{|l|}{ T stage } \\
\hline $\mathrm{TI}$ & 7423 & 57.1 & 97 & & & \\
\hline $\mathrm{T} 2$ & 4321 & 33.2 & 89 & & & \\
\hline T3 & 692 & 5.3 & 79 & & & \\
\hline $\mathrm{T} 4$ & 571 & 4.4 & 65 & $<0.001$ & $<0.001$ & $1.628-1.919$ \\
\hline \multicolumn{7}{|l|}{ Lymph nodes status } \\
\hline Without lymph nodes resection & 3016 & 23.2 & 91 & & & \\
\hline No metastatic lymph node & 6945 & 53.4 & 96 & & & \\
\hline With metastatic lymph nodes & 3046 & 23.4 & 83 & $<0.001$ & 0.520 & $0.999-1.003$ \\
\hline \multicolumn{7}{|l|}{ Grade } \\
\hline GI & 3369 & 25.9 & 98 & & & \\
\hline G2 & 6115 & 47 & 95 & & & \\
\hline G3 & 3447 & 26.7 & 82 & & & \\
\hline G4 & 46 & 0.4 & 89 & $<0.001$ & $<0.001$ & $1.605-2.094$ \\
\hline \multicolumn{7}{|l|}{ Distance metastasis } \\
\hline Mo & 12803 & 98.4 & 93 & & & \\
\hline MI & 204 & 1.6 & 42 & $<0.001$ & $<0.001$ & $4.517-7.296$ \\
\hline \multicolumn{7}{|l|}{ Surgical approach ${ }^{\mathrm{a}}$} \\
\hline Partial mastectomy & 7939 & 61.0 & 95 & & & \\
\hline Simply total mastectomy & 2763 & 21.2 & 90 & & & \\
\hline Radical mastectomy & 2305 & 17.8 & 83 & $<0.001$ & $<0.001$ & I.109-1.375 \\
\hline \multicolumn{7}{|l|}{ Radiotherapy } \\
\hline Yes & 4374 & 33.6 & 95 & & & \\
\hline No & 8287 & 63.7 & 90 & & & \\
\hline Unknown & 346 & 2.7 & 93 & $<0.001$ & $<0.001$ & $0.520-0.731$ \\
\hline \multicolumn{7}{|l|}{ ER status } \\
\hline Positive & 11207 & 86.2 & 94 & & & \\
\hline Negative & 1797 & 13.8 & 78 & & & \\
\hline Border & 3 & 0.02 & 60 & $<0.001$ & 0.001 & $1.151-1.725$ \\
\hline \multicolumn{7}{|l|}{ PR status } \\
\hline Positive & 9609 & 73.9 & 95 & & & \\
\hline Negative & 3369 & 25.9 & 83 & & & \\
\hline Border & 29 & 0.2 & 71 & $<0.001$ & $<0.001$ & $1.581-2.315$ \\
\hline \multicolumn{7}{|l|}{ HER-2 status } \\
\hline Positive & 1240 & 9.5 & 85 & & & \\
\hline Negative & || $45 \mid$ & 88 & 93 & & & \\
\hline Border & 316 & 2.4 & 85 & $<0.001$ & 0.152 & $0.953-1.363$ \\
\hline
\end{tabular}

Note: aThe "local" approach includes partial mastectomy, and the "radical" approach includes subcutaneous mastectomy, modified radical mastectomy, radical mastectomy and extended radical mastectomy.

Abbreviations: ER, estrogen receptor; PR, progesterone receptor; HER-2, human epidermal growth factor receptor-2. 
A

Points

Grade

Tumor size

Age

Distant metastasis

Race

Surgical approach

Radiotherapy

ER status

Total points

1-year cancer-specific survival

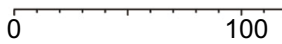

White

Others

Black

Simply total mastectomy

Partial Radical mastectomy

mastectomy Unknown

With Without

Positive Negative

Negative

Positive Border

2-year cancer-specific survival

3-year cancer-specific survival

B $\stackrel{\infty}{\circ}$

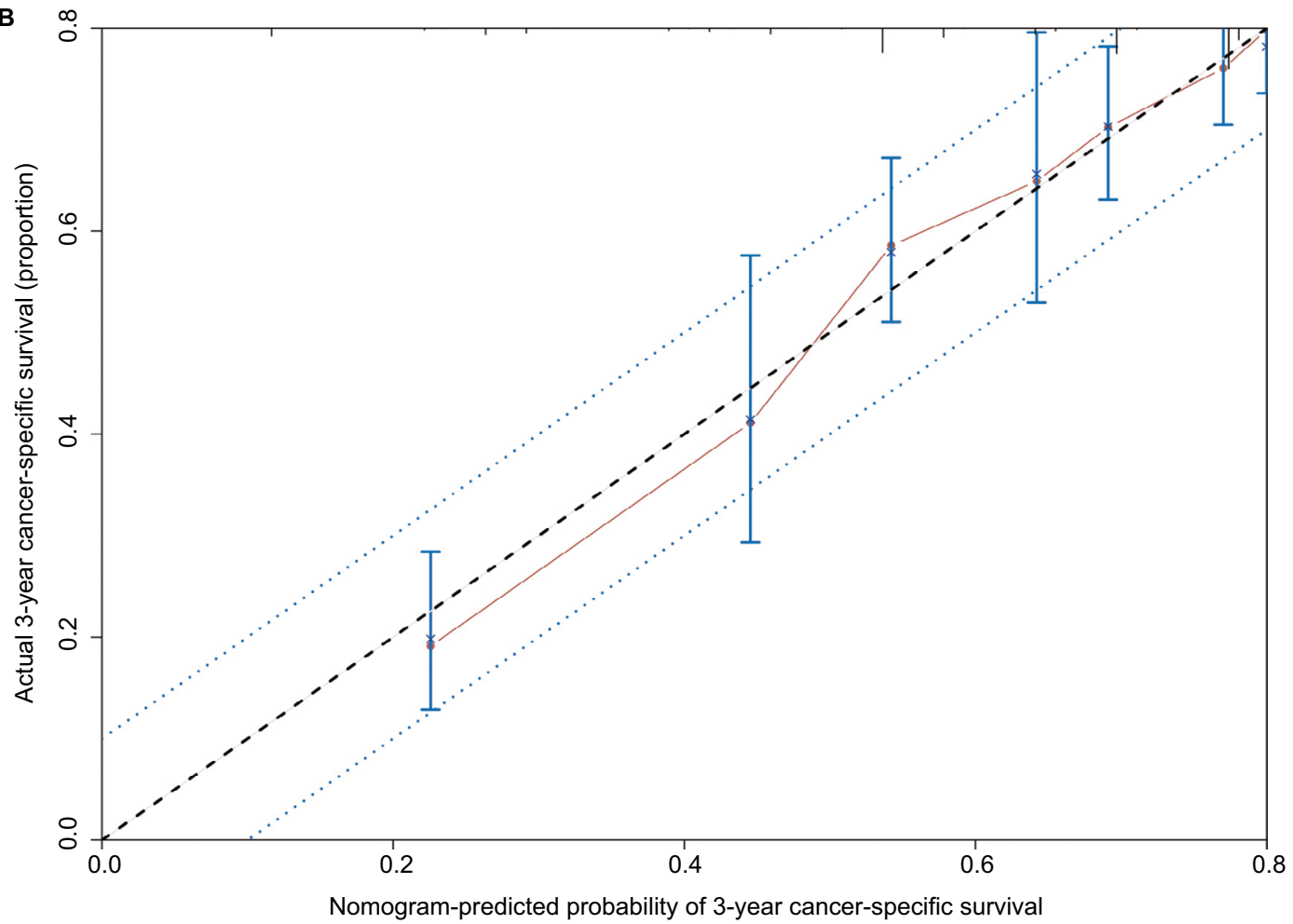

Figure 2 The prediction and calibration of the nomogram.

Notes: (A) A nomogram was used to predict I- to 3-year cancer-specific survival rates using nine independent prognostic characteristics. (B) The nomogram was calibrated for elderly patients with breast cancer. The nomogram predicted the probability of cancer-specific survival, which was plotted on the $x$-axis, and the actual cancer-specific survival rate, which was plotted on the $y$-axis. We measured $95 \%$ Cls using the Kaplan-Meier analysis. All predictions were within the 10\% margin of error (indicated by the dotted blue line). 
three surgical approaches according to differences in tumor size and the use of radiotherapy. The results indicated that a localized surgical approach resulted in better cancer-specific survival regardless of whether or not the patients were treated with radiotherapy. Moreover, localized surgery also resulted in better cancer-specific survival when the tumor size was $<50 \mathrm{~mm}$ in diameter. In addition, in patients in whom the tumor size larger was $<50 \mathrm{~mm}$, cancer-specific survival was not worse in treatment using a localized surgical approach than an extended surgical approach (Figure 3).

In order to reduce the bias caused by the retrospective analysis, a propensity score analysis was performed. After patients who underwent local and radical surgical approaches were matched, 5068 pair patients were included in the validation set. Figure S2 shows a jitter plot of the data for matched and unmatched patients as well as the corresponding distri- butions of propensity score values. We used a matched data set for further analysis, and the results indicated that while localized surgery resulted in better cancer-specific survival when tumor size was $<50 \mathrm{~mm}$, radiotherapy was required to improve cancer-specific survival (Figure S3).

\section{Discussion}

Patients with breast cancer who are older than 80 years represent a unique segment of the population. Which treatment strategies are best in this type of patient remains to be determined. When choosing a strategy, we should consider life expectancy, the potential benefits of treatment, the patient's goals for treatment and the potential risks associated with treatment, including its effects on function and quality of life. ${ }^{1}$ Therefore, cancer-specific survival may be a more appropriate factor for evaluating treatment strategies and other prognostic indexes.
A

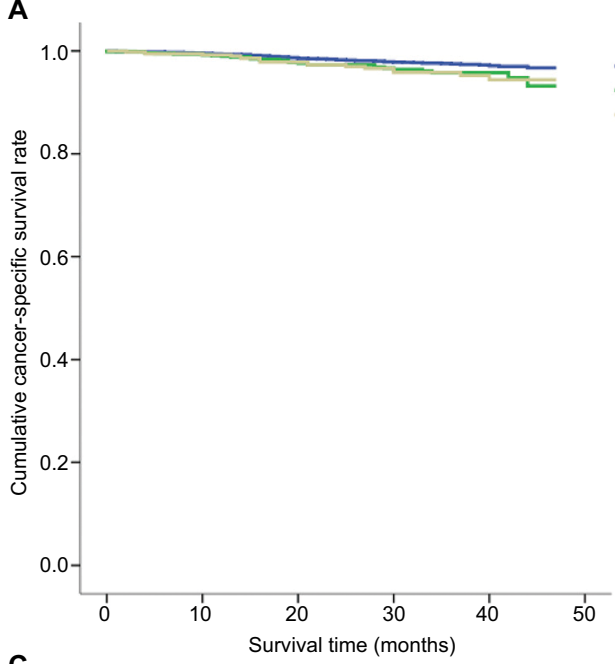

C

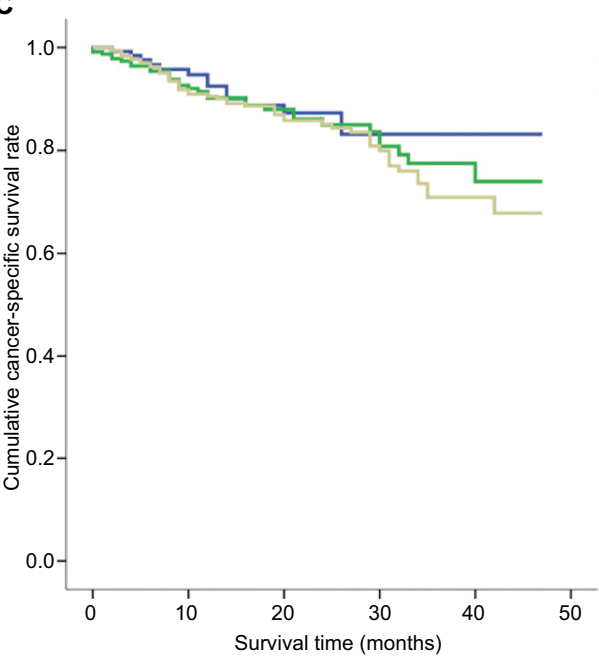

Surgical approach $\rightarrow$ Partila mastectomy $\rightarrow$ Simply total mastectomy Radical mastectomy

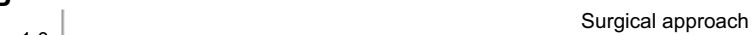

$1.0-1$ Purgical approach
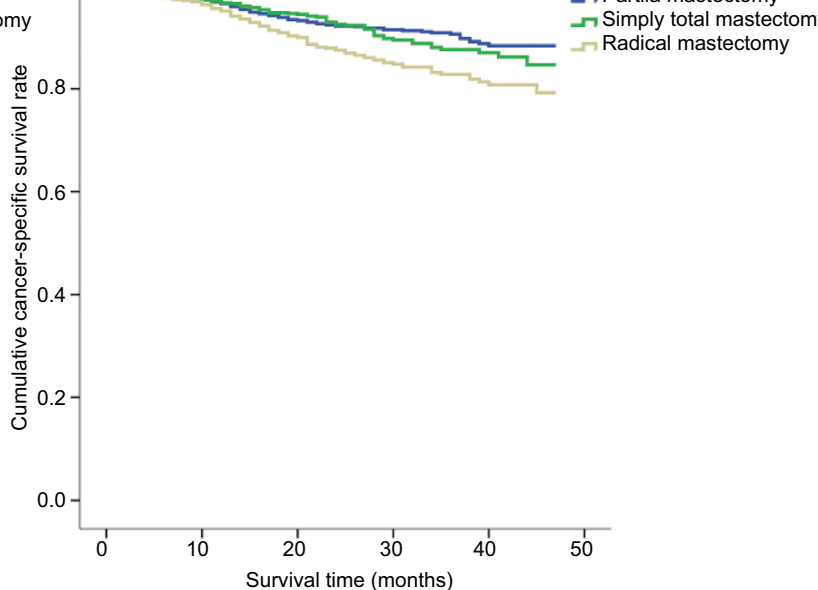

D

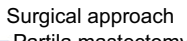
$\rightarrow$ Partila mastectomy $\rightarrow$ Simply total mastectom Radical mastectomy

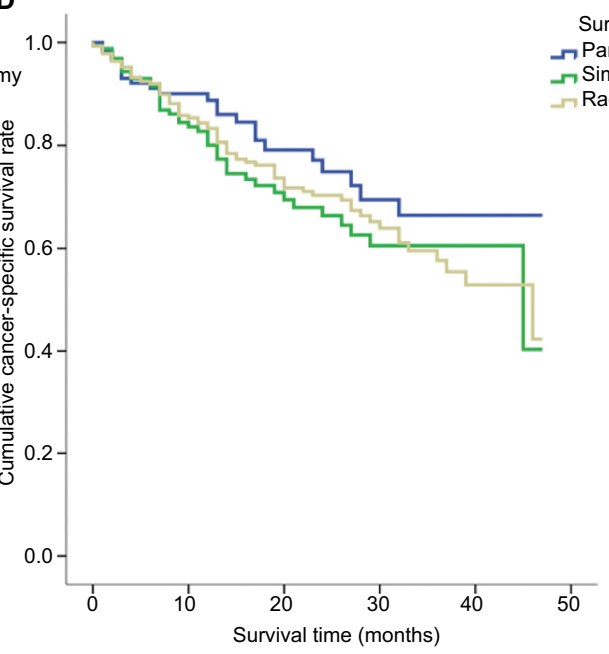

Figure 3 Cancer-specific survival according to the included surgical approach categories.

Notes: The results are stratified by tumor size. (A) AJCC TI, (B) AJCC T2, (C) AJCC T3 and (D) AJCC T4.

Abbreviation: AJCC, American Joint Committee on Cancer. 
Other studies have found that patients who are 80 years or older tolerate surgery well with low complication rates. ${ }^{2,3}$ These data indicate that although complications occurred in $37.1 \%$ of this cohort, $31.4 \%$ of all complications were minor. In addition, in elderly patients with breast cancer who underwent surgery, perioperative mortality was zero. However, in our study, the results indicated that a localized surgical approach was not worse than extended surgery for estimating cancer-specific survival. At the same time, localized surgery did not substantially change the shape of the breast and resulted in fewer traumas. Therefore, patients were more likely to accept this type of surgical procedure. Furthermore, another study has supported the idea that most older women could be considered candidates for breast preservation. $^{7}$

In patients with breast cancer tumors $<3 \mathrm{~cm}$ in size and negative lymph nodes and who are ER and/or PR positive and HER-2 negative, breast-conserving therapy and endocrine therapy without breast radiation are viable options. No undergoing radiation had no effect on overall survival but may be associated with the rate of breast cancer recurrence. ${ }^{8,9}$ In our study, we found that radiotherapy improved cancer-specific survival and overall survival in elderly breast cancer patients. In elderly breast cancer patients who might not need radiotherapy (eg, those with a tumor size $<2 \mathrm{~cm}$, negative HER-2 expression and positive ER/PR expression), radiation also provided some benefits to cancer-specific survival and overall survival. However, we should acknowledge that radiotherapy might increase the risk of cardiovascular incidents, for which the HR increment ranged from 1.5 to $2.4 .^{10}$ In patients with cardiovascular-related severe diseases, a strategy involving radiotherapy requires careful consideration. The HR quoted is for mediastinal radiation, which is rarely done these days. The heart dose is usually reduced with deep inspiration breath-holding technique.

The nomogram prediction scheme may provide a more accurate and personalized survival estimate in elderly breast cancer patients. This prognostic nomogram directly quantifies patient survival risk based on variant prognostic factors without forming risk groups, a strategy that resulted in more favorable $C$ and AIC indexes than were achieved using the AJCC TNM classification. ${ }^{11}$ Cancer-specific prognostic factors are different in elderly patients than in younger patients. Our nomogram can also be used to indicate which factors most influence cancer-specific survival, which might give clinicians important insight when treating these patients.

Our study has several limitations. First, the data for perioperative chemotherapy were not available for all patients in the SEER database. Additionally, the benefits of chemotherapy in elderly breast cancer patients were controversial. Some studies have suggested that elderly patients who undergo adjuvant therapy have worse outcomes, while others endorse its use. ${ }^{12,13}$ Second, endocrine therapy was also not available in the database. These two factors are critical prognostic factors in patients with breast cancer. However, the absence of data regarding the use of chemotherapy or endocrine therapy did not significantly influence the choice of surgical approach. In addition, the use of chemotherapy or endocrine therapy in breast cancer is based on the stage of the disease and its molecular subtype. Our study was based on a large sample size of $\sim 17000$ patients who were recruited during the past 5 years. This may have reduced potential bias in the analysis. In addition, we used the propensity score method to solve the problem of imbalance in baseline characteristics between different treatment groups. The propensity score represents the probability of assigning a patient to a treatment, and it can be calculated using a fitted model. Propensity matching is nonparametric, and the two-step procedure used in causal effect estimation is considered doubly robust in that if either the propensity score matching or the parametric model is correct, the causal estimates should be consistent. ${ }^{14,15}$ Furthermore, applying a stratified adjusted survival analysis would make the analysis more accurate when comparing the relationship between potential prognosis factors and the 5-year overall survival rate. Third, the usage of trastuzumab was unclear, and this might have affected the results for HER-2-positive patients. Fourth, as a retrospective study of the SEER database, we have to acknowledge that there were some limitations, but this database included a large population. Furthermore, we used propensity score analysis in order to decrease some bias caused by the missing items. In elderly patients, the cancer-specific survival might be a good factor to predict the effects of the treatment strategy. In the nomogram prediction model, every factor was allocated a score, so we omit the stage information and include the distant metastasis information.

\section{Conclusion}

A localized surgical approach might be a better choice in elderly breast cancer patients. However, radiotherapy was needed to improve cancer-specific survival and overall survival in these patients. In addition, we developed a prognostic nomogram that could be used to directly quantify patient risk based on variant prognostic factors without requiring the formation of risk groups, and this approach was more favorable for estimating cancer-specific survival. 


\section{Acknowledgments}

We thank Jianguo Lai for his assistance during data analysis. This research was not supported by any specific grant from a funding agency in the public, commercial, or not-for-profit sectors. version of the manuscript.

\section{Disclosure}

The authors report no conflicts of interest in this work.

\section{References}

1. Shachar SS, Hurria A, Muss HB. Breast cancer in women older than 80 years. J Oncol Pract. 2016;12(2):123-132.

2. Weiss A, Noorbaksh A, Tokin C, Chang D, Blair SL. Hormone receptornegative breast cancer: undertreatment of patients over 80. Ann Surg Oncol. 2013;20(10):3274-3278

3. Bao J, Yu K-D, Jiang Y-Z, Shao Z-M, Di G-H. The effect of laterality and primary tumor site on cancer-specific mortality in breast cancer: a SEER Population-Based Study. PLoS One. 2014;9(4) e94815-e94818.

4. Yancik R, Wesley MN, Ries LA, Havlik RJ, Edwards BK, Yates JW. Effect of age and comorbidity in postmenopausal breast cancer patients aged 55 years and older. JAMA. 2001;285(7):885-892.

5. Rosenbaum PR, Rubin DB. Reducing bias in observational studies using subclassification on the propensity score. J Am Stat Assoc. 1984;79(387):516-524.
6. Zhang Z. Propensity score method: a non-parametric technique to reduce model dependence. Ann Transl Med. 2017;5(1):7.

7. Cheung SYA, Evans ND, Chappell MJ, Godfrey KR, Smith PJ, Errington RJ. Exploration of the intercellular heterogeneity of topotecan uptake into human breast cancer cells through compartmental modelling. Math Biosci. 2008;213(2):119-134.

8. Balducci L. Treatment of breast cancer in women older than 80 years is a complex task. J Oncol Pract. 2016;12(2):133-134.

9. Hughes KS, Schnaper LA, Bellon JR, et al. Lumpectomy plus tamoxifen with or without irradiation in women age 70 years or older with early breast cancer: long-term follow-up of CALGB 9343. J Clin Oncol. 2013;31(19):2382-2387.

10. Nolan MT, Russell DJ, Marwick TH. Long-term risk of heart failure and myocardial dysfunction after thoracic radiotherapy: a systematic review. Can J Cardiol. 2016;32(7):908-920.

11. Zhou Z, Zhang H, Xu Z, Li W, Dang C, Song Y. Nomogram predicted survival of patients with adenocarcinoma of esophagogastric junction. World J Surg Oncol. 2015;13(1):197.

12. Janssen-Heijnen ML, Houterman S, Lemmens VE, Louwman MW, Maas HA, Coebergh JW. Prognostic impact of increasing age and comorbidity in cancer patients: a population-based approach. Crit Rev Oncol Hematol. 2005;55(3):231-240.

13. Schonberg MA, Marcantonio ER, Li D, Silliman RA, Ngo L, Mccarthy EP. Breast cancer among the oldest old: tumor characteristics, treatment choices, and survival. J Clin Oncol. 2010;28(12):2038-2045.

14. Ho DE, Stuart EA. Matching as nonparametric preprocessing for reducing model dependence in parametric causal inference. Polit Anal. 2007;15(3):199-236.

15. Keller B, Tipton E. Propensity score analysis in R: a software review. J Educ Behav Stat. 2016;41(3):326-348. 


\section{Supplementary materials}

In order to validate the prognostic prediction model, the propensity score analysis was used. After the matching of local and radical surgical approaches, 5068 paired patients were included the validation set. Overall survival and cancerspecific survival were significantly better in patients who underwent surgery than in those who did not $(P<0.001$, Figure S1A and S1B), as shown in Figure S1. In addition, overall survival and cancer-specific survival were significantly better in patients who received radiotherapy than in those who did not $(P<0.001$, Figure S1C and S1D). Figure S2 shows the jittered plot of the matched and unmatched observations, as well as their distribution on propensity score values. At the same time, the results indicated that localized surgery also had better cancer-specific overall survival when the tumor size was less than $50 \mathrm{~mm}$ (Figure S3).
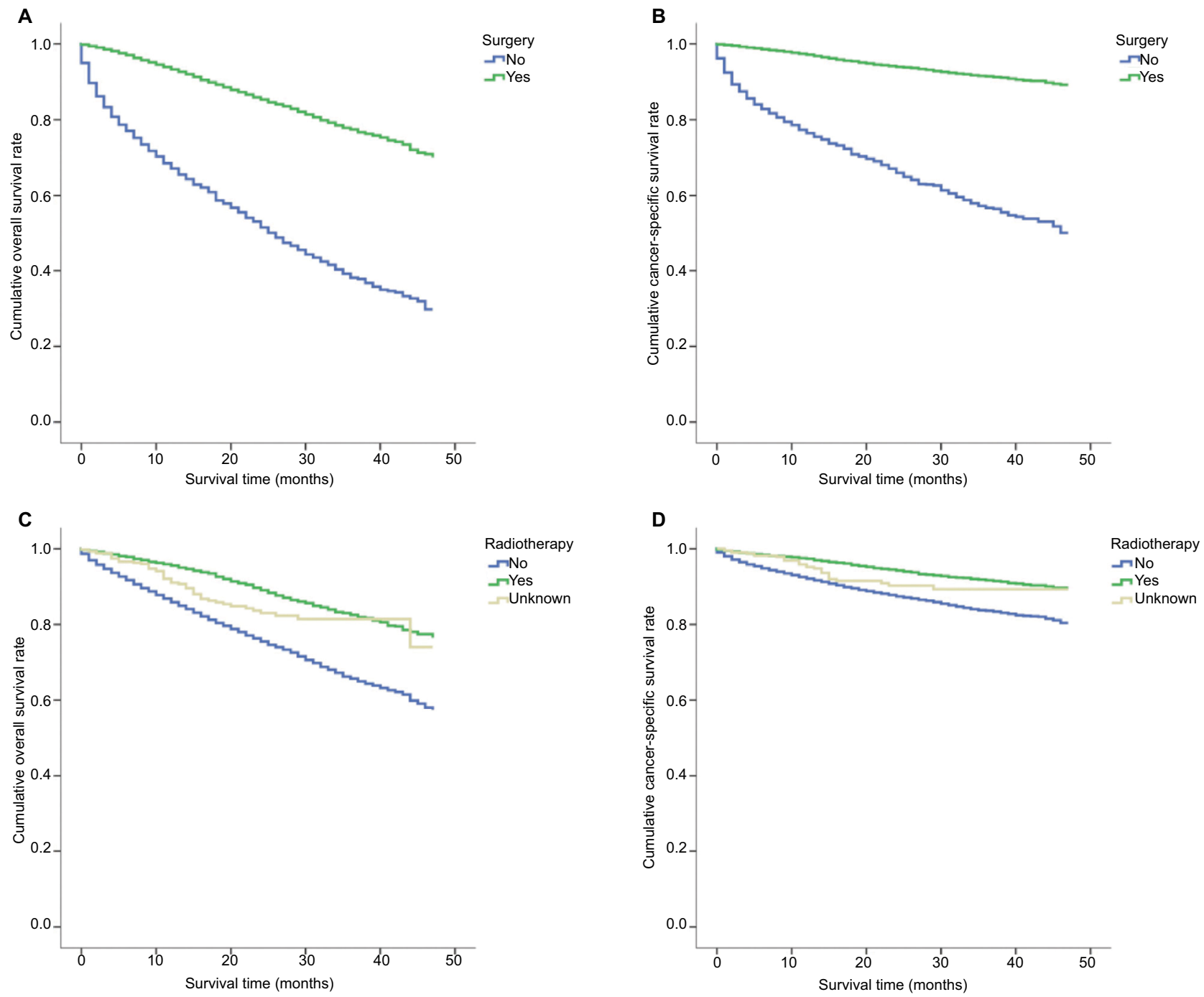

Figure SI The effects of surgery and radiotherapy for survival of elderly breast cancer patients. 
Distribution of propensity scores

Unmatched treatment units

Matched treatment units

$\circ$

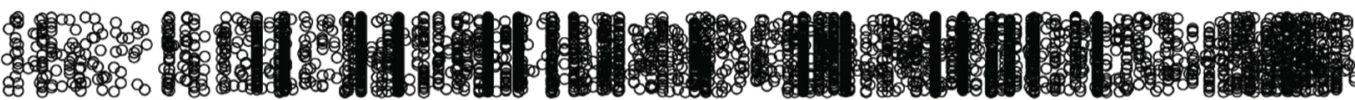

Matched control units

$\circ$ 每

Unmatched control units

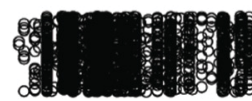

0.0

0.2

0.4

0.6

0.8

1.0

Propensity scores

Figure S2 The jittered plot of the matched and unmatched observations and their distribution on propensity score values. 


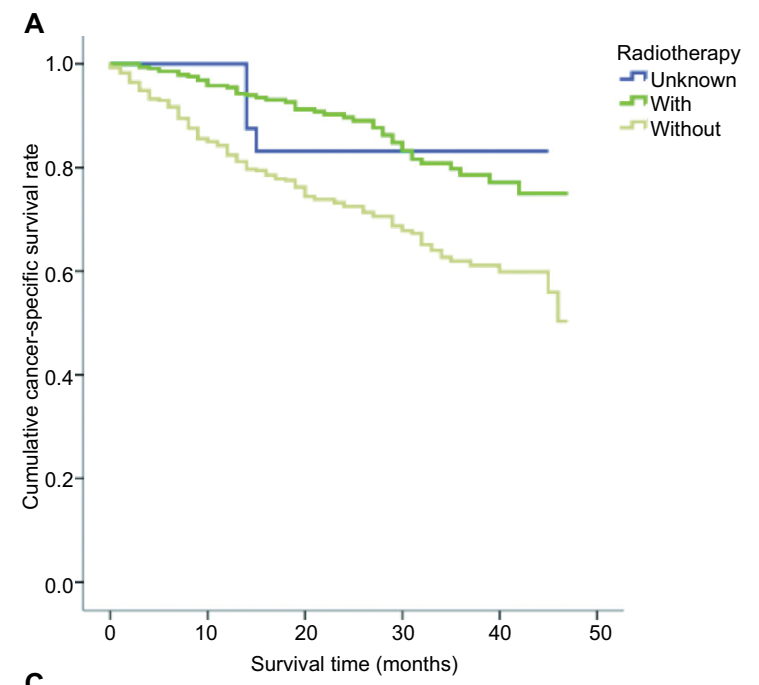

C

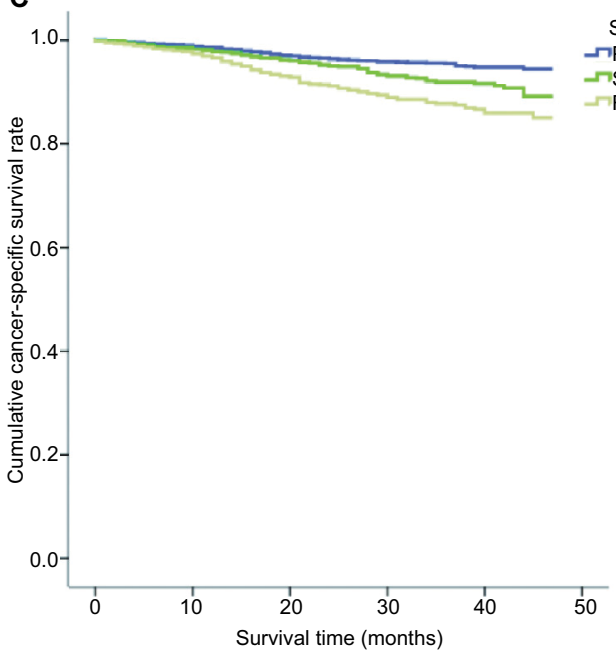

B

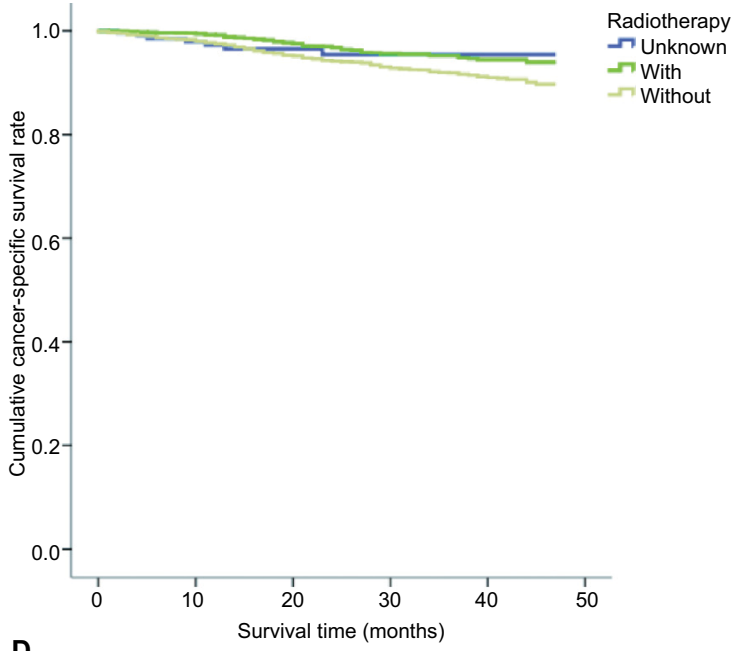

D

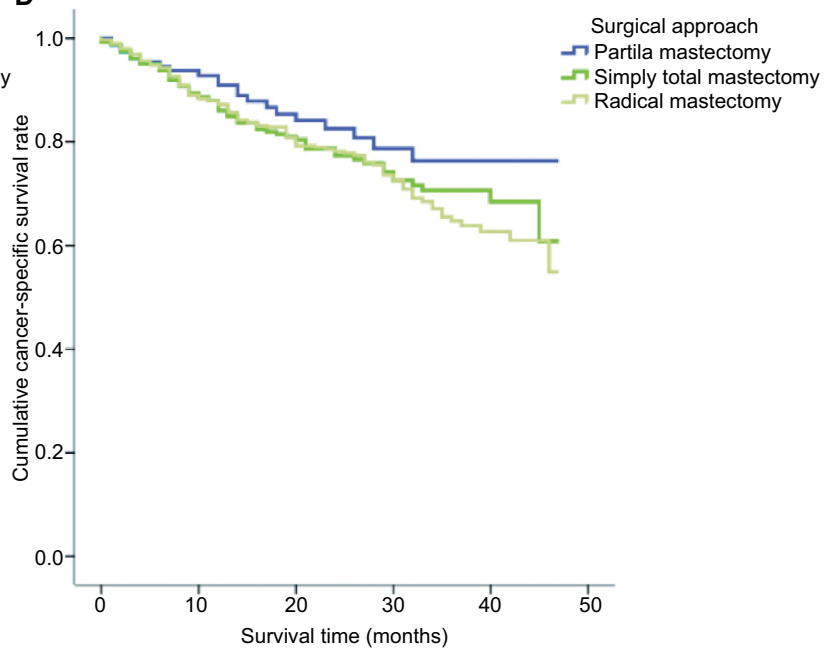

Figure S3 The cancer-specific overall survival of different treatments after PSA matching. (A) tumor less than $50 \mathrm{~mm}$. (B) tumor greater than $50 \mathrm{~mm}$, (C) tumor less than $50 \mathrm{~mm}$, (D) tumor greater than $50 \mathrm{~mm}$.

Abbreviation: PSA, propensity score analysis.

Cancer Management and Research

\section{Publish your work in this journal}

Cancer Management and Research is an international, peer-reviewed open access journal focusing on cancer research and the optimal use of preventative and integrated treatment interventions to achieve improved outcomes, enhanced survival and quality of life for the cancer patient. The manuscript management system is completely online and includes
Dovepress

a very quick and fair peer-review system, which is all easy to use. Visit $\mathrm{http}: / / \mathrm{www}$.dovepress.com/testimonials.php to read real quotes from published authors. 